\title{
Löfgren syndrome in acute sarcoidosis
}

\author{
Alexandra P. Saltman MD, Bindee Kuriya MD SM \\ Cite as: CMAJ 2017 October 2;189:E1230. doi: 10.1503/cmaj.170547
}

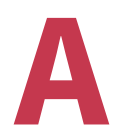

61-year-old woman of Italian descent with a history of hypertension and gastroesophageal reflux disease presented to the emergency department with a two-week history of pain, erythema, swelling and restricted range of motion of her left ankle. The patient was prescribed cephalexin for possible cellulitis and celecoxib for possible crystalline arthritis; however, there was no improvement. To rule out septic or crystalline arthritis, aspiration of her left ankle was attempted but did not yield any synovial fluid.

The patient's symptoms progressed over four days to involve her right ankle, left hand, both knees, right elbow and distal right arm. She reported fevers and night sweats. Physical examination was notable for restricted range of motion of her ankles because of pain, with surrounding warmth, erythema and edema (periarthritis) that extended to the proximal shins (Figure 1A). She also had tender erythematous nodules on her forearms (Figure 1B) and hands. The patient's erythrocyte sedimentation rate was 95 (normal range less than 20) $\mathrm{mm} / \mathrm{h}$ and level of serum C-reactive protein was 14760 (normal range less than 476) nmol/L. Results from routine hematology and biochemistry tests were normal, and results for work-up for infectious disease were negative. Radiography of the chest showed bilateral adenopathy of the hilar lymph nodes without interstitial markings (Appendix 1, available at www. cmaj.ca/lookup/suppl/doi:10.1503/cmaj.170547/-/DC1). These findings were confirmed by computed tomography of the chest.

We diagnosed Löfgren syndrome based on the presence of erythema nodosum, bilateral hilar adenopathy and polyarthritis. ${ }^{1}$ This constellation of symptoms has a $95 \%$ diagnostic specificity for this acute variant of sarcoidosis. ${ }^{2}$ The differential diagnosis is broad, including atypical mycobacterial and fungal infections, drug-induced serum sickness, lymphoma, reactive arthritis, crystalline arthritis and vasculitis.

We prescribed prednisone (50 mg daily taken orally), given our patient's failure to respond to first-line treatment with nonsteroidal anti-inflammatory drugs. ${ }^{3}$ Within 72 hours, she had substantial reduction in inflammatory signs and symptoms. At one month, she had fully recovered and was no longer receiving treatment. Most patients (more than $80 \%$ ) with Löfgren syndrome have a monophasic illness with an excellent prognosis. ${ }^{2}$

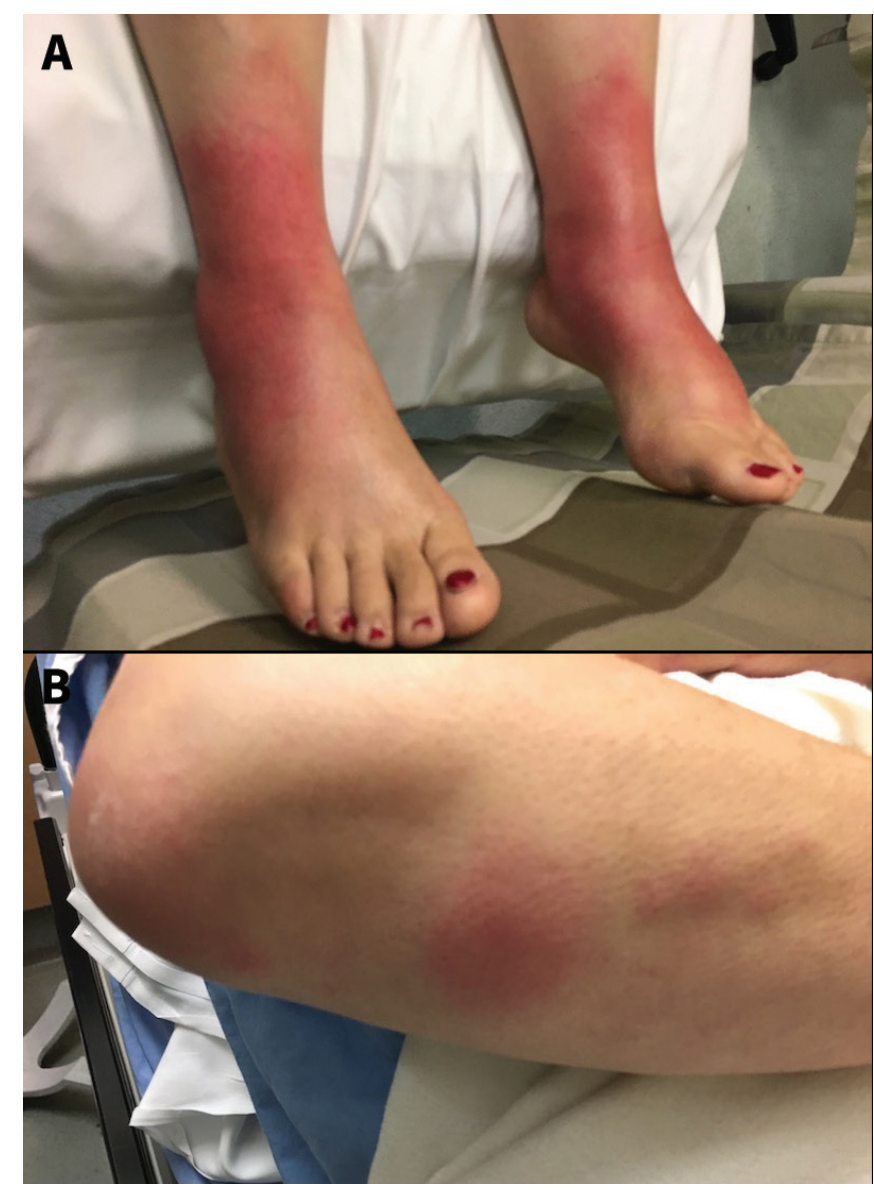

Figure 1: A 61-year-old woman with (A) juxta-articular erythema and edema of both ankles characteristic of periarthritis, and multiple raised erythematous and tender nodules located over the extensor surfaces of her hands and (B) forearms that were consistent with erythema nodosum.

\section{References}

1. Lofgren S, Lundback H. The bilateral hilar lymphoma syndrome; a study of the relation to tuberculosis and sarcoidosis in 212 cases. Acta Med Scand 1952;142:265-73.

2. O'Regan A, Berman JS. Sarcoidosis. Ann Int Med 2012;156:ITC5-1-15.

3. Abril A, Cohen MD. Rheumatologic manifestations of sarcoidosis. Curr Opin Rheumatol 2004;16:51-5.

\section{Competing interests: None declared.}

This article has been peer reviewed.

The authors have obtained patient consent.
Affiliation: Division of Rheumatology, Department of Medicine, Faculty of Medicine, University of Toronto, Toronto, Ont.
Correspondence to: Alexandra Saltman, alexandra.saltman@gmail.com 